\title{
artigo
}

\section{Desafios e potencialidade da atuação da equipe multiprofissional na atenção primária em saúde}

\author{
Challenges and potentiality of the multiprofessional team's performance in primary health care \\ Retos y potencialidad del desempeño del equipo multiprofesional en atención primaria de salud
}

\section{RESUMO}

Objetivo: Descrever a percepção da equipe multiprofissional do Núcleo de Ampliado de Saúde da Família e Atenção Básica (NASF-AB) sobre sua atuação. Método: Estudo qualitativo, observacional, analítico, realizado com 32 profissionais de 11 equipes do NASF-AB de Belém (PA). Foi realizada entrevista semiestruturada que versava sobre a atuação, atribuições, barreiras, potencialidades da equipe multiprofissional. Os dados foram analisados pelo software ALCEST. Resultados: Foram identificadas 3 classes: "dificuldades e barreiras" como a precariedade de recursos, falta de segurança, pouca capacitação; "potencialidades", primordialmente o trabalho multiprofissional; e, "atuação da equipe" na prevenção e promoção à saúde. Conclusão: Embora os profissionais do NASF-AB reconheçam as potencialidades da atuação da equipe multidisciplinar e que o trabalho esteja em sua maioria pautado na prevenção e promoção à saúde as barreiras sempre se fazem presentes, dificultando a sua plena atuação na atenção primária em saúde.

DESCRITORES: Sistema Único de Saúde; Atenção Primária à Saúde; Estratégia Saúde da Família; Profissional da Saúde; Equipe Multiprofissional.

\section{ABSTRACT}

Purpose: To describe the perception of the NASF-AB multiprofessional team about their performance. MethodoQualitative and observational analytical study, carried out with 32 professionals from 11 NASF-AB teams in Belém (PA). A semi-structured interview was carried out that dealt with the performance, duties, guidelines, barriers and potential of the multidisciplinary team. The data were analyzed by the ALCEST software. Results: Three classes were identified: "difficulties and barriers" such as lack of resources, lack of security, little training; "Potentialities", primarily multidisciplinary work; and, "team performance" in health prevention and promotion. Conclusion: Although NASF-AB professionals recognize the potential of the multidisciplinary team and that their work is mostly based on prevention and health promotion, barriers are always present, hindering their full performance in PHC.

DESCRIPTORS: Unified Health System; Primary Health Care; Family Health Strategy; Health professional; Multiprofessional team.

\section{RESUMEN}

Objetivo: Describir la percepción del equipo multiprofesional NASF-AB sobre su desempeño. Metodología: Estudio analítico cualitativo y observacional, realizado con 32 profesionales de 11 equipos NASF-AB en Belém (PA). Se realizó una entrevista semiestructurada que abordó el desempeño, deberes, lineamientos, barreras, potencialidades del equipo multidisciplinario. Los datos se analizaron utilizando el software ALCEST. Resultados: Se identificaron tres clases: "dificultades y barreras" como falta de recursos, falta de seguridad, poca formación; "Potencialidades", principalmente trabajo multidisciplinario; y "desempeño en equipo" en prevención y promoción de la salud. Conclusión: Si bien los profesionales de NASF-AB reconocen el potencial del equipo multidisciplinario y que el trabajo se basa mayoritariamente en la prevención y promoción de la salud, las barreras siempre están presentes dificultando su pleno desempeño en la APS.

DESCRIPTORES: Sistema Único de Salud; Primeros auxilios; Estrategia de salud familiar; Profesional de la salud; Equipo multiprofesional.

RECEBIDO EM: 30/10/2020 APROVADO EM: 27/11/2020

\section{Suellen Cristinne Macedo de Sá}

Fisioterapeuta e pós-graduanda em Musculação Terapêutica e Fisiologia do Exercício. Centro Universitário do Estado do Pará, Curso de Fisioterapia.

ORCID: 0000-0002-7362-8099 


\section{Evenly Arlinda Costa dos Santos}

Fisioterapeuta e pós-graduada em Fisioterapia hospitalar e Gestão Hospitalar e dos Serviços de Saúde. Centro Universitário do Estado do Pará, Curso de Fisioterapia.

ORCID: 0000-0003-0214-5257

\section{Nathália Brito da Silva}

Fisioterapeuta e pós-graduanda em Fisioterapia em Traumatologia, Ortopedia e Desportiva. Centro Universitário do Estado do Pará, Curso de Fisioterapia.

ORCID: 0000-0002-6039-1483

\section{Brenda Stefany de Campos Chaves}

Fisioterapeuta Residente em Neurologia e pós-graduanda em Fisioterapia em Terapia Intensiva. Centro Universitário do Estado do Pará, Curso de Fisioterapia.

ORCID: 0000-0001-7566-2541

\section{Soanne Chyara Soares Lira}

Fisioterapeuta e Mestre em Neurociências e Biologia Celular. Centro Universitário do Estado do Pará, Curso de Fisioterapia; Universidade Estadual do Pará, Curso de Fisioterapia.; Universidade Estadual do Pará, Curso de Fisioterapia.

ORCID: 0000-0001-5272-9993

\section{INTRODUÇÃO}

$\mathbf{N}$ o processo de priorização do Sistema Único de Saúde - SUS, o fortalecimento da Atenção Primária à Saúde (APS) tem sido realizado para ampliar a cobertura de atenção à saúde da família, atingir a equidade e melhorar a qualidade de atenção à saúde. Os atores deste processo compõem tanto a equipe de Estratégia de Saúde da Família (ESF) que desde 1994 consolidam a porta de entrada do SUS, quanto o Núcleo de Ampliado de Saúde da Família e Atenção Básica (NASF-AB) que desde 2008 atuam primordialmente com bases dos princípios da integralidade, interdisciplinariedade, resolubilidade, seguindo as diretrizes da Política Nacional de Atenção Básica (PNAB) 1,2,3,4,5.

Com intuito de apoiar a ESF, o NASF-AB é composto por equipe multiprofissional, que de modo histórico tem sido vinculada à atenção secundária e terciária à saúde como fonoaudiólogo, fisioterapeuta, nutricionista, terapeuta ocupacional, dentre outros. A seleção dos profissionais se dá conforme a demanda de cada região abrangida pelas equipes da $\mathrm{ESF}^{3}$. Várias são as atividades a serem desenvolvidas por esses profissionais, e toda proposta deve ser compartilhada com a equipe - seja ela de atendimentos individuais, atenção domiciliar a acamados, atendimentos em grupo, oficinas de educação em saúde ou estudos de caso, entre outras ${ }^{6,7}$.
O modelo implantado ao mesmo tempo que aumenta o acesso e resolubilidade, impõe questões relacionadas à sua prática, como atividades individuais ou coletivas, atendimento de prevenção/ promoção ou assistencial, modelo de assistência matricial, capacitação profissional, além da complexidade que o trabalho multiprofissional e intersetorial impelem ${ }^{8,9}$.

O NASF-AB é um programa inovador e ainda com muitos desafios para implantar uma proposta que objetiva a integralidade, o trabalho multiprofissional e até intersetorial. Há necessidade de reorganização, descentralização para que se tenham ações prioritárias de prevenção e promoção da saúde. Inúmeros estudos verificam a percepção, atuação, avanços, desafios de uma classe profissional específica no NASF-AB. Esta pesquisa propõe estudar a percepção da equipe multiprofissional do NASF-AB sobre a sua atuação, em relação às suas atribuições e da equipe, e verificar potencialidades e barreiras da atuação do NASF-AB.

\section{MÉTODO}

Trata-se de um estudo qualitativo e observacional do tipo analítico que foi realizado após a aprovação do Comitê de Ética e Pesquisa (CEP) do Centro Universitário do Estado do Pará, sob CAAE: 85946318.2.0000.5169/2018.
Participaram 32 profissionais de diversas categorias dos 11 NASF-AB tipo II de Belém (PA) no período de agosto a dezembro de 2018. Após a assinatura do Termo de Consentimento Livre e Esclarecido foram utilizados dois instrumentos de coleta de dados: questionário para os dados sociodemográficos analisados por estatística descritiva e entrevista semiestruturada para a atuação da equipe multiprofissional.

A entrevista semiestruturada foi composta por 7 questões as quais abordam a relevância do trabalho multiprofissional, atribuições da equipe, interação com a ESF, se a atuação ocorre de acordo com o que é preconizado, barreiras e potencialidades encontradas, além de desafios e metas da equipe, a qual foi gravada de maneira individual e presencial, por um único entrevistador.

Para análise dos dados, os áudios foram transcritos e analisados no programa Alceste (do francês "Analyse Lexicale par Contexte d'un Ensemble de Segment de Texte", e em português "Análise Lexical Contextual de um Conjunto de Segmentos de Texto"), no qual os dados transcritos foram primeiramente adaptados a um corpus, em que palavras tidas como termo próprio foram transcritas para leitura adequada do programa, por exemplo, "promoção_à saúde”, "estratégia_de_saúde_da_família”, "equipe_multiprofissional”. $\mathrm{Na}$ sequência o programa realizou a parametrização se- 
guindo algumas etapas: análise do vocabulário, definição das unidades de contexto, classificação das unidades de contexto e definição das classes de significados, ${ }^{10}$.

A partir das classes de significados estabelecidas pelo software foi realizada a nomeação dessas classes, e após, elencado comentários das próprias entrevistas transcritas (tanto por sugestão do próprio programa quanto pelo corpus geral) que representem a unidade apresentada pelo software. Para a identificação da fala, mantendo o sigilo dos entrevistados, cada categoria profissional foi citada seguida do número do NASF_AB de atuação.

\section{RESULTADOS E DISCUSSÃO}

\section{Caracterização sociodemográfica dos profissionais do NASF-AB}

A idade média dos participantes foi de 35,43 anos $( \pm 8,31)$, sendo que os profissionais atuantes do NASF-AB, em sua maioria, eram indivíduos adultos jovens; $71,87 \%$ dos participantes eram do sexo feminino e $28,12 \%$ do sexo masculino; as profissões que participaram foram: Educador Físico (3), Psicólogo (7), Terapeuta Ocupacional (4), Nutricionista (1), Fonoaudiólogo (3), Fisioterapeuta (10) e Assistente Social (4); a média do tempo de formados destes foi de 8,96 anos $( \pm 5,35)$ e o tempo de serviço atual apresentou em média 3,38 anos $( \pm 1,22)$.

\section{Classificação das classes}

A análise qualitativa, utilizou $65 \%$ das unidades do corpus, que foram qualificadas segundo o nível de relevância, sendo então as unidades classificadas divididas em três classes (FIGURA 1). A classe 1 foi a mais específica, seu vocabulário foi mais homogêneo, representando 57\% das unidades classificadas, sendo caracterizada por palavras como: barreira, dificuldade, falta; a classe 2, que representa $24 \%$ das unidades de texto classificadas, tem palavras significativas: importância, relevância, potencialidade; e, a classe 3, que representa $19 \%$ das unidades de texto classificadas, tem como palavras significativas: atuação, prevenção, qualidade de vida, promoção à saúde.

\section{Classe 1 - Barreiras/ Dificuldades}

Representa o que a equipe enfrenta na sua atuação, como as dificuldades referentes a infraestrutura, insegurança com a violência, déficits na comunicação multiprofissional e formação profissional, falta de compreensão dos usuários e equipe multiprofissional sobre as atribuições do NASF-AB.

Os aspectos da infraestrutura, que envolve tanto o transporte como os materiais, estão destacados abaixo:

"As regióes distantes que a gente atende, às vezes não é possivel a gen-
Figura 1: Porcentagem de representação das classes do corpus analisados. Belém / PA (2018).

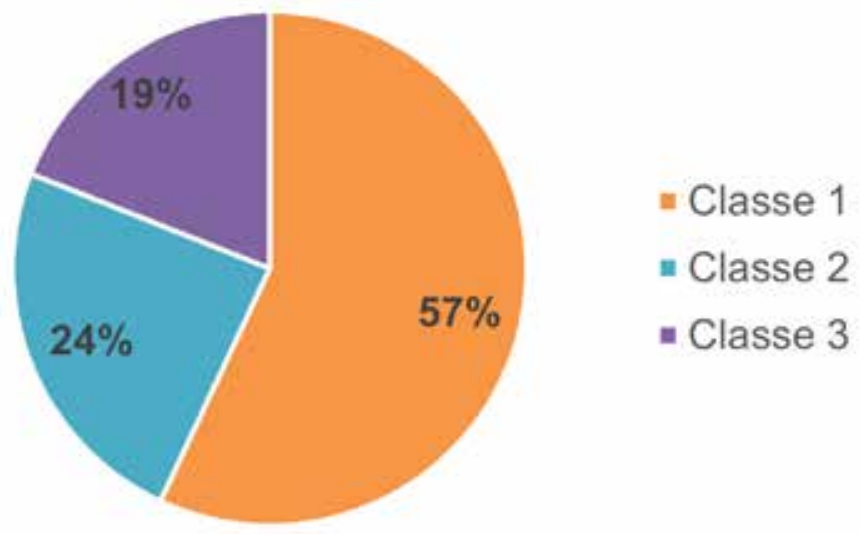

te fazer mais de 2 ou 3 visitas por dia, realmente fica inviável, então se houvesse um transporte, ajudaria muito." (Fisioterapeuta - 5).

"As barreiras são essas: falta de utensílios, materiais para atividades com os grupos, com a comunidade, coisas simples como bola, bambolê, cone." (Fisioterapeuta - 8).

A dificuldade de transporte é recorrente em diversos NASF-AB, ou pela ausência ou disponibilidade parcial do transporte ${ }^{11,12,13}$. As visitas domiciliares são uma das ações previstas na PNAB4 para a assistência integral a qual o NASF-AB se propõe. Quanto aos materiais, é prática comum que os profissionais façam uso dos seus materiais pessoais ou seus próprios recursos para suprir a falta, algo que pode estar correlacionado à deficiência da gestão ${ }^{11}$.

A insegurança é outro quesito abordado:

"Às vezes a gente tem medo de ir pra área porque a gente vivencia isso no dia a dia, a questão de assalto, violência, então isso precisa ser melhorado." (Fisioterapeuta - 10).

"Pra gente entrar na comunidade, às vezes, a questão do tráfico, né? Que tem que pedir autorização pro traficante, a questão da violência." (Educador Físico 7).

A periculosidade de algumas áreas geográficas na realização do trabalho no NAS$\mathrm{F}-\mathrm{AB}$, geralmente associadas ao tráfico de drogas, é um grande obstáculo para o desempenho do trabalho ${ }^{11}$. Embora o vínculo com a comunidade minimize os efeitos da violência para com os profissionais, o processo de trabalho em que estão inseridos torna-se "adoecedor", pois enfrentam situações de grande vulnerabilidade ${ }^{14}$.

Outros aspectos de funcionamento da própria equipe também podem ser barreiras para a atuação:

"Mas antes disso, já passou pela ESF, já teve o pedido e pela falta de comunicação, a gente não conseguiu atender." (Educador Físico - 10).

"A principal barreira que a gente

Fonte: Pesquisa de campo, 2018. 
encontra é a questão dessa comunicação, dessa interação entre os profissionais, é a parceria com alguns profissionais da ESF porque o NASF não trabalha sozinho." (Fisioterapeuta - 7).

A qualidade das relações estabelecidas com as ESF, envolvendo a comunicação, é um importante aspecto para reorganização do processo de trabalho entre as equipes. No entanto, há diferenças entre as duas equipes que poderiam dificultar esse processo, tais como a formação e a experiência dos profissionais; a dinâmica prevista do trabalho; a facilidade e/ ou dificuldade em compartilhar algumas ações ${ }^{15}$. Além disso, o NASF-AB não se configura como porta de entrada do sistema de saúde e deve atuar de forma articulada e integrada às $\mathrm{ESF}^{2,4}$. Como consequência, a falta de comunicação interfere no atendimento mais integral e resolutivo que o SUS propõ $\mathrm{e}^{16}$.

Além da comunicação, a falta de compreensão dos usuários e equipe da ESF sobre as atribuições do NASF-AB, são descritas a seguir:

"A barreira maior que a gente encontra é da aceitação ainda na comunidade. A gente tá lá pra promover a saúde das pessoas e as pessoas ainda não têm essa consciência." (Psicólogo - 11).

"A barreira maior que tu tem é de mudar a cultura do atendimento, do remédio, do curativo, tu entender a prevenção." (Fisioterapeuta - 6).

"Então a gente tá sempre expondo, treinando e capacitando os agentes comunitários de saúde para esse entendimento do que é o nosso trabalho, pra que eles possam compreender também... é... o desafio grande é tentar passar pra eles o trabalho da nossa equipe e o trabalho individual de cada um." (Assistente Social - 8).

Há falta de compreensão tanto das equipes e gestores envolvidos como dos usuários $^{15}$, sobre a PNAB e da atuação do NASF-AB, e aos diferentes interesses rela- cionados aos distintos modelos de atenção em saúde vigentes. A proposta de trabalho do núcleo tem provocado resistências em algumas equipes de ESF, que demonstram um distanciamento no campo de traba1 ho ${ }^{3,4}$. Com isso, o NASF-AB requer das equipes ESF modificações na forma de trabalho, que, por vezes estão centralizadas em práticas assistenciais, curativas e individuais.

Outra dificuldade encontrada e discutida é o déficit na formação profissional:

"Vocês vão perceber que vieram daquela formação muito individualista, principalmente na saúde, né? $E$ chega aqui não é nada individual, não deve ser pelo menos". (Terapeuta Ocupacional - 11)

A formação profissional pautada em modelo biomédico ainda é fator que dificulta a efetivação das ações na APS. Muitos profissionais não denotam um perfil de formação adequado aos objetivos do NASF-AB e transparecem algumas lacunas na formaçãa ${ }^{18,19}$. Ter abordagem integral perpassa pela capacidade de contrastar o paradigma biomédico, enfatizar a prevenção e a promoção da qualidade de vida, e atuar com equipe multiprofissional.

\section{Classe 2 - Potencialidades}

Os profissionais ressaltam como principal potencialidade o trabalho multiprofissional, a partir de diversos prismas. Este capaz de garantir a resolubilidade e a integralidade da atenção e saúde, como exposto abaixo:

"[...] o trabalho multiprofissional é uma potencialidade, você ter profissionais de diferentes áreas atuando em conjunto, isso facilita muito você trabalhar uma problemática, porque aí são vários olhares [...] vão te auxiliar, de repente no teu encaminhamento." (Assistente Social - 8).

O objetivo do processo de trabalho do NASF-AB é qualificar e dar auxílio ao trabalho desenvolvido pelas ESF, agindo de maneira participativa e cooperando para suplantar a atenção fragmentada que ainda funciona no modelo de saúde vigente, contribuindo para a estruturação de redes de atenção à saúde $(\mathrm{RAS})^{20}$.

"A gente tem condiçôes de compreender a problemática, a demanda que chega, como um todo, dentro de um olhar multiprofissional. Então, a gente consegue ter uma percepção melhor, então pra estudo de caso, pra gente poder trabalhar essa demanda que vem." (Assistente Social - 8).

A participação dos profissionais do NASF-AB, como um grande diferencial que promove maior agilidade e resolutividade através de reuniões de discussões de casos, viabilizando as ações em saúde desses profissionais na distribuição dos saberes e fazeres assumindo maior segurança nas atividades adotadas, para gerar intervenções conjuntas, valorizando a discussão de casos e seus desdobramentos junto a ESF ${ }^{21}$.

"Açôes na escola, ações de prevenção, ações de saúde, ações de fortalecimento é... Educação e saúde, atendimento individual, visita domiciliar pra pacientes acamados." (Terapeuta Ocupacional - 11).

O processo de trabalho da equipe multiprofissional ajuda a suprir a demanda da comunidade reduzindo danos e agravos, promovendo a saúde ${ }^{19}$. Para a atuação em diversos cenários a utilização das ferramentas do NASF são essenciais: Apoio Matricial, Clínica Ampliada, o Projeto Terapêutico Singular (PTS), o Projeto de Saúde no Território (PST) e a Pactuação do Apoio². Observa-se que as ações descritas nas falas acima trazem consigo a prática das ferramentas, que oferece subsídios para a promoção da qualidade de vida da população.

\section{Classe 3 - Atuação da equipe}

Exposição dos pontos relevantes acerca da assistência integral oferecida pela equipe, assim como as atividades planejadas e executadas de forma multiprofissional para atingir a prevenção, são fatores que podem ser observados na fala abaixo:

"[...] É trabalhar a prevenção e a orientação, para que os usuários não 
venham adquirir, por exemplo, uma hipertensão e diabetes, algum problema no caso de fisio, algum problema locomotor, cinesiológico [...]". (Fisioterapeuta - 1).

A integralidade pode ser considerada a principal diretriz a ser praticada pelo NASF-AB, sendo compreendida nas práticas das ações de promoção e prevenção de saúde ${ }^{1,2}$. Ações estas que ampliam a resolubilidade das ações da atenção primária e ainda atuar como um facilitador do acesso dos usuários à assistência em saúde mais complexa e abrangente ${ }^{15}$.

São destacados, atribuições e serviços ofertados pelos profissionais, e o planejamento das ações junto à equipe ESF:

"A gente tem como atribuição [...] fazer atendimento coletivo, atendimento domiciliar, dar palestras, a gente faz a promoção e prevenção da saúde através das orientações [...]". (Psicólogo - 10)

"As atribuições do NASF preconizam a prevenção. Com grupos terapêuticos, oficinas terapêuticas cognitivas, da memória e vários temas de palestra para o fim de prevenir". (Psicóloga-2).

Uma das atribuições do NASF-AB é desenvolver coletivamente, com vistas à intersetorialidade, ações que se integrem a outras políticas sociais, como educação, esporte, cultura, trabalho, lazer, entre outras ${ }^{1,2}$. Estas são de sua responsabilidade e executadas de forma articulada com as ESF, como o desenvolvimento do projeto de saúde na área, planejamentos, apoio aos grupos, trabalhos educativos, de inclusão social e ações junto aos espaços públicos com enfoque na promoção e prevenção em saúde.

$\mathrm{O}$ apoio matricial e demais questões sobre prevenção também são mencionados.

"A gente faz o apoio matricial, além disso, a busca ativa daqueles pacientes que necessitam de orientação. Nosso trabalho principal na verdade é prevenção e promoção à saúde. Não é 0 atendimento terciário. $E$ a atenção primária realmen- te. É orientar e promover saúde". (Fisioterapeuta - 5).

$\mathrm{O}$ apoio matricial destaca-se como estratégia do NASF-AB, que garante a assistência especializada às ESF. Tal assistência pode ocorrer de duas formas: oferta assistencialista em saúde especializada ao indivíduo, e por meio do apoio técnico pedagógico ${ }^{1,2,15}$. Através do acordo entre as equipes, a assistência especializada é ofertada ao usuário nas situações clínicas de maior abrangência e, sobre o apoio técnico pedagógico, sua assistência consiste em uma ação ligada à troca de saberes e de práticas/experiências.

Acerca da busca ativa de um perfil específico de usuários, favorece as ofertas de serviços voltadas para as necessidades de cada território, levando-se em consideração os conhecimentos/informações e as percepções de inúmeras categorias profissionais ${ }^{15}$.

Sobre outras atribuições da equipe, é mencionado:

"[...] Na UMS o trabalho se da com os pacientes que já apresentam alguma alteração, algum atraso, né? No desenvolvimento ou que tenham algum exame suspeito que elas precisam encaminhar pra gente". (Fisioterapeuta -9).

Apesar de o NASF-AB integrar a APS, o mesmo não se constitui em porta de entrada da RAS e sua ação é iniciada somente após a identificação das necessidades do território adscrito às $\mathrm{ESF}^{1,2}$. Todavia, a falta de acesso a serviços especializados pode induzir ao desempenho equivocado, estabelecendo propostas de atendimentos ambulatoriais, o que não compete ao NASF-AB ${ }^{24}$. Este seria um retrocesso relacionado ao modelo de atenção à saúde vigente que não oferece resolubilidade e dificulta a implantação plena da $\mathrm{PNAB}^{4,25}$.

\section{Interrelação entre as classes}

Compreende-se que cada classe possui suas particularidades, palavras e objetivos distintos, no entanto, os aspectos pontuados nas classes de palavras evidenciados pe- los profissionais, também se interligam com pontos em comum, condizendo que, embora o NASF-AB trabalhe de forma interdisciplinar e integrada à ESF, ainda encontra limitações para realiza-lo e além de ter a promoção em saúde como uma atribuição e com uma diversidade em potencialidades, as barreiras ainda se fazem presentes, justificando que por ser um programa em implantação, ainda encontra muitas limitações (Figura 2).

\section{Figura 2: Interrelação entre as classes 1, 2 e 3 em coordenadas. Belém / PA (2018).}

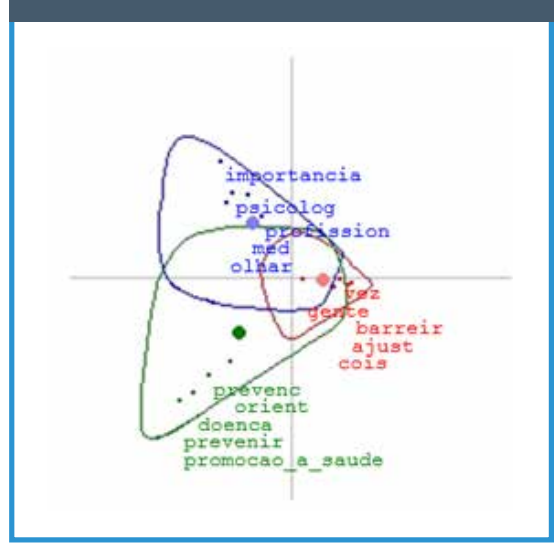

Fonte: Pesquisa de campo, 2018

\section{CONCLUSÃO}

Conclui-se que existem muitos desafios que precisam ser transpostos para otimizar o trabalho da equipe multiprofissional do NASF-AB como a precariedade da infraestrutura, insegurança frente a violência, déficits na comunicação multiprofissional, deficiência na formação profissional para atuar na APS e falta de compreensão dos usuários e equipe multiprofissional da ESF sobre as atribuições do NASF-AB.

Mesmo com estes desafios, a equipe multiprofissional percebe as potencialidades de sua atuação como planejamento de ações de modo integrado e contextualizado com as demandas da população adscrita, intervenção tanto de forma individual quanto na coletividade, otimização do funcionamento das RAS, qualidade na continuidade do cuidado aos usuários, ou seja, da integralidade.

Por fim, para reduzir as barreiras e oti- 
mizar as potencialidades, é necessário que estudos como este busquem investigar a percepção de outros atores, como a gestão para garantia do trabalho multiprofissional no âmbito da APS, ou os usuários de utilizam cuidados da equipe multiprofis- sional do NASF-AB, para que uma visão amplificada possa garantir a efetivação da integralidade em saúde.

\section{REFERÊNCIAS}

1. BRASIL, Ministério da Saúde. Cadernos de Atenção Básica: Diretrizes do NASF. Brasília, DF. 2009. Disponível em: <http:// bvsms.saude.gov.br/bvs/publicacoes/caderno_atencao_basica_diretrizes_NASF.pdf >. Acesso em: 10 jan. 2020.

2. BRASIL, Ministério da Saúde. Cadernos de Atenção Básica: Núcleo de Apoio à Saúde da Família- volume 1: Ferramentas para a gestão e para do trabalho cotidiano. Brasília, DF. 2014. Disponível em: <http://bvsms.saude.gov.br/bvs/publicacoes/nucleo_apoio_saude_familia_cab39.pdf>. Acesso em: 10 jan. 2020.

3. Maia FES, Moura ELR, Madeiros EC, Carvalho RRP, Silva SAL, Santos GR. A importância da inclusão do profissional fisioterapeuta na atenção básica de saúde. Rev Fac Ciênc Méd Sorocaba [serial on the internet]. 2015 [cited 2020 Out 20];17(3):110-5. Available from: http://revistas.pucsp.br/index.php/RFCMS/article/view/16292/pdf

4. BRASIL. Ministério da Saúde. Portaria n 2.436 de 21 de setembro de 2017. Brasília: Diário Oficial da República, 2017. Disponível em: <http://bvsms.saude.gov.br/bvs/saudelegis/gm/2017/ prt2436_22_09_2017.html>. Acesso em: 10 jan. 2020.

5. Cruz AG da, Monteiro E, Borges CJ, Pelazza BB, Silva LA da, Paula CR de, Maia LG. Nova política nacional de atenção básica: percepções dos gestores municipais do sistema único de saúde. SaudColetiv (Barueri) [Internet]. $11^{\circ}$ de maio de 2020 [citado $30^{\circ}$ de outubro de 2020];9(49):1599 - 1604. Disponivel em: http://revistas.mpmcomunicacao.com.br/index.php/saudecoletiva/article/view/131

6. Oliveira ICO, Rocha RM, Cutolo LRA. Algumas palavras sobre o nasf: relatando uma experiência acadêmica. Rev Bras Educ Méd [Internet]. 2012 [cited 2020 Out 20];36(4):574-80.

7. Silva ATCS, Aguiar ME, Winck K, Rodrigues KGW, Sato ME, Grisi SJFE, et al. Núcleos de Apoio à Saúde da Família: desafios e potencialidades na visão dos profissionais da Atenção Primária no Município de São Paulo, Brasil. Cad Saude Publica. 2012;28(11):207684. DOI:10.1590/S0102-311X2012001100007

8. Melo EA et al. Dez anos dos Núcleos de Apoio à Saúde da Família (Nasf): problematizando alguns desafios. Saúde em Debate [online]. 2018; 42(1): 328-340. Disponivel em: <https://doi. org/10.1590/0103-11042018S122>. ISSN 2358-2898. https:// doi.org/10.1590/0103-11042018S122.

9. Souza TS e Medina MG. Nasf: fragmentação ou integração do trabalho em saúde na APS?. Saúde em Debate [online]. 2018; 42(2): 145158. Disponível em: <https://doi.org/10.1590/0103-11042018S210>. ISSN 2358-2898. https://doi.org/10.1590/0103-11042018S210.

10. Reinert M. Postures énonciatives et mondes lexicaux stabilisés en analyse statistique de discours. Langage et société. 2007; 121-122( 3 ):189-202.

11. Santos WTM; Mandelbaum BPH. Entre o potencial e o precário: a inserção in (tensa) de profissionais da psicologia nos NASF. Barbarói. 2006; 48: 168-184.

12. Souza GA. A Percepção dos Agentes Comunitários de Saúde quanto ao Núcleo de Apoio à Saúde da Família em Águas Lindas-Ananindeua-Pará. 2016. Monografia (Especialista em Saúde da Família) - Universidade do Estado do Pará, Belém, 2016.

13. Vasconcelos PB. Plano de intervenção para aumentar a adesão dos idosos ao tratamento de hipertensão arterial no PSF Baguari-município de Governador Valadares/MG. 2017. Monografia (Especialista em Estratégia Saúde da Família) - Universidade Federal de Minas Gerais, Governador Valadares, 2017.

14. Leite DF, Nascimento DDG, Oliveira MAC. Qualidade de vida no trabalho de profissionais do NASF no município de São Paulo. Physis: Revista de Saúde Coletiva, 2014; 24(2):507-525.

15. Gonçalves RMA. et al. Estudo do trabalho em Núcleos de Apoio à Saúde da Família (NASF), São Paulo, Brasil. Revista Brasileira de Saúde Ocupacional. 2015; 40(131):59-74.

16. Nascimento CMB. et al. Configurações do processo de trabalho em núcleos de apoio à saúde da família e o cuidado integral. Trabalho, Educação e Saúde, 2018; 16(3):1.135-1.156.

17. Aciole GG e Oliveira DKS. Perceptions of users and professionals of the family health about the Family Health Support Center. Saúde em Debate. 2017; 41(115):1090-1101.

18. Santos S, Benedetti T, Sousa T., Fonseca S. Apoio Matricial ea atuação do Profissional deEducação Física do Núcleo de Apoio à Saúde da Familia. Revista Brasileira de Atividade Física \& Saúde. 2017; 22(1):54-65.

19. Brito RFSLV, Leal MDCP, Aragão JA, Maia VLLB, Lago EC, Figueiredo LS. O idoso na estratégia saúde da familia: atuação do enfermeiro durante o envelhecimento ativo. Revista Interdisciplinar. 2015; 8(4):99-108.

20. Vendruscolo Carine, Trindade Letícia de Lima, Maffissoni André Lucas, Martini Jussara Gue, Silva Filho Cláudio Claudino da, Sandri Juliana Vieira de Araujo. Implicação do processo de formação e educação permanente para atuação interprofissional. Rev. Bras. Enferm. [Internet]. 2020 [cited 2020 Oct 20]; 73 ( 2 ): e20180359. Available from: http:/www.scielo.br/scielo. php?script=sci_arttext\&pid=S0034-71672020000200181\&lng=en. Epub Mar 30, 2020. https:/doi.org/10.1590/0034-7167-2018-0359.

21. De Souza MC, Bomfim AS, Souza JN, Batista TF. Fisioterapia e Núcleo de Apoio à Saúde da Família: conhecimento, ferramentas e desafios. O Mundo da Saúde. 2013:176.

22. Moreira TDNF, Martins CL, Feuerwerker LCM, Schraiber LB. A construção do cuidado: o atendimento às situações de violência doméstica por equipes de Saúde da Familia. Saúde e Sociedade. 2014; 23(3):814-827.

23. Bezerra MIC, Lima MJMR, Lima YCP. A visita domiciliar como ferramenta de cuidado da fisioterapia na estratégia saúde da família. SANARE-Revista de Políticas Públicas. 2015; 14(1).

24. Cunha GT, Campos GWDS. Apoio matricial e atenção primária em saúde. Saúde e Sociedade. 2011; 20(4):961-970.

25. Melo EA, Mendonça MHMD, Oliveira JRD, Andrade GCLD. Mudanças na Política Nacional de Atenção Básica: entre retrocessos e desafios. Saúde em Debate. 2018; 42:38-51. 